\title{
Quantum dot-based molecular imaging of cancer cell growth using a clone formation assay
}

\author{
XIA-FEI GENG ${ }^{1}$, MIN FANG ${ }^{1}$, SHAO-PING LIU ${ }^{2}$ and YAN LI ${ }^{1}$ \\ ${ }^{1}$ Department of Oncology, Hubei Key Laboratory of Tumor Biological Behaviors and Hubei Cancer Clinical Study Center; \\ ${ }^{2}$ Medical Research Center, Zhongnan Hospital of Wuhan University, \\ Wuhan, Hubei 430071, P.R. China
}

Received June 9, 2015; Accepted July 25, 2016

DOI: $10.3892 / \mathrm{mmr} .2016 .5632$

\begin{abstract}
This aim of the present study was to investigate clonal growth behavior and analyze the proliferation characteristics of cancer cells. The MCF-7 human breast cancer cell line, SW480 human colon cancer cell line and SGC7901 human gastric cancer cell line were selected to investigate the morphology of cell clones. Quantum dot-based molecular targeted imaging techniques (which stained pan-cytokeratin in the cytoplasm green and Ki67 in the cell nucleus yellow or red) were used to investigate the clone formation rate, cell morphology, discrete tendency, and Ki67 expression and distribution in clones. From the cell clone formation assay, the MCF-7, SW480 and SGC7901 cells were observed to form clones on days 6,8 and 12 of cell culture, respectively. These three types of cells had heterogeneous morphology, large nuclear:cytoplasmic ratios, and conspicuous pathological mitotic features. The cells at the clone periphery formed multiple pseudopodium. In certain clones, cancer cells at the borderline were separated from the central cell clusters or presented a discrete tendency. With quantum dot-based molecular targeted imaging techniques, cells with strong Ki67 expression were predominantly shown to be distributed at the clone periphery, or concentrated on one side of the clones. In conclusion, cancer cell clones showed asymmetric growth behavior, and Ki67 was widely expressed in clones of these three cell lines, with strong expression around the clones, or aggregated at one side. Cell clone formation assay based on quantum dots molecular imaging offered a novel method to study the proliferative features of cancer cells, thus providing a further insight into tumor biology.
\end{abstract}

Correspondence to: Professor Yan Li, Department of Oncology, Hubei Key Laboratory of Tumor Biological Behaviors and Hubei Cancer Clinical Study Center, Zhongnan Hospital of Wuhan University, 169 Donghu Road, Wuchang, Wuhan, Hubei 430071, P.R. China

E-mail: liyansd2@163.com

Key words: cell clone formation, quantum dots, Ki67

\section{Introduction}

Uncontrolled proliferation is a hallmark of cancer cell behavior (1), with morphological manifestations in vitro in cell culture and in vivo during tumor proliferation, invasion and metastasis. During in vitro cell culture, cell proliferation lead to the formation of cell clones. The clone formation rate and morphological characteristics can reflect the biological behavior of cancer cells (2-4).

$\mathrm{Ki67}$, a cell-cycle-related non-histone and a common predictive index of cell proliferation, is expressed during all cell cycle phases except for the $\mathrm{G}_{0}$ phase (5), particularly in breast cancer, stomach cancer, colon cancer, lung cancer, liver cancer, lymphoma and other malignant tumors (6,7). Quantum dots (QDs), are novel fluorescent nano-particles with unique properties (8-10), including broad and continuous excitation spectra, narrow and symmetrical emission spectra, strong brightness, high photostability and a long fluorescence lifetime. The QD-based molecular probe technique has a distinct advantage for investigating the characteristics of tumor growth and invasion compared with fluorescent proteins or organic dyes, including size tunable light emission, enhanced signal brightness and resistance to photo bleaching $(11,12)$. Cell clone formation assays are an important technical method for detecting cancer cell proliferation potential, invasiveness and susceptibility to hazardous factors (13). The present study focused on three common cancer cell lines, MCF-7 breast cancer cells, SW480 colon cancer cells and SGC7901 gastric cancer cells. These cells were used to detect the distribution and expression of Ki67 after the cell clone formation assay using the QD-based molecular probe technique. This in vitro study was designed to simulate the early stages of tumor formation, in order to investigate cancer cell growth and the proliferation.

\section{Materials and methods}

Cell culture. The MCF-7, SW480 and SGC7901 cells were obtained from the stock from the Medical Research Center, Zhongnan Hospital of Wuhan University (Wuhan, China). MCF-7 cells were cultured in Dulbecco's modified Eagle's medium (DMEM)/high glucose (HyClone, Logan, UT, USA) supplemented with $10 \%$ fetal bovine serum (FBS; Zhejiang 
Tianhang Biotechnology Co., Ltd., Huzhou, China) and $1 \%$ penicillin/streptomycin (HyClone). SW480 cells and SGC7901 cells were cultured in RPMI-1640 (HyClone) supplemented with $10 \%$ FBS and $1 \%$ penicillin/streptomycin. Cells were incubated in a humidified atmosphere of $95 \%$ air and $5 \% \mathrm{CO}_{2}$ at a constant temperature of $37^{\circ} \mathrm{C}$.

Cell clone formation assay. Tumor cells were digested by $0.25 \%$ trypsin/ $0.02 \%$ EDTA solution at the logarithmic phase to make a single-cell suspension with culture medium. Then, a cell counting chamber wsa sued to calculate the number of cells in a $10 \mu \mathrm{l}$ single-cell suspension under an inverted microscope. The cells were then delivered into six-well culture plates containing a sterile glass cover-slip in each well, with $500-1,000$ cells added to each well. The medium was refreshed every 3 days until cell clones could be observed with the naked eye.

Immunostaining. This study followed the previously described QD-based fluorescent immunostaining protocols for histological and cytological studies with slight modifications (14-17). Briefly, the cells on the glass coverslips were fixed with $4 \%$ paraformaldehyde after washing with phosphate-buffered saline (PBS, pH 7.2-7.4; Bioyear Beijing Medical System Co., Ltd., Beijing, China). Bovine serum albumin (BSA, Biosharp, Wuhan, China), serving as a blocking buffer, was added and the cells were cultured for $30 \mathrm{~min}$ at $37^{\circ} \mathrm{C}$. Then, cells were stained with monoclonal mouse anti-pan-cytokeratin (CK) (cat. no. JY-0077, Jiayuan Quantum Dots Co. Ltd., Wuhan, China, dilution 1:25 for MCF-7 cells, 1:50 for SW480 cells, 1:25 for SGC7901 cells) and rabbit anti-Ki67 (cat. no. JY-0047, Jiayuan Quantum Dots Co. Ltd.; dilution 1:100 for MCF-7 cells, 1:250 for SW480 cells, 1:100 for SGC7901 cells). The secondary antibodies were QDs-525 goat $\mathrm{F}\left(\mathrm{ab}^{\prime}\right) 2$ anti-mouse IgG conjugate (Invitrogen; Thermo Fisher Scientific, Inc., Waltham, MA, USA; dilution 1:200, emitting green light at wavelength $525 \mathrm{~nm}$ ), QDs-585 goat $\mathrm{F}$ (ab') 2 anti-rabbit IgG conjugate (cat. no. Q-11411MP; Invitrogen; Thermo Fisher Scientific, Inc.; dilution 1:200, emitting yellow light at wavelength $585 \mathrm{~nm}$ ) and QDs-605 goat F (ab') 2 anti-rabbit IgG conjugate (cat. no. QM 605; Jiayuan Quantum Dots Co. Ltd.; dilution 1:100, emitting red light at wavelength $605 \mathrm{~nm}$ ). primary antibodies at $4^{\circ} \mathrm{C}$ overnight and then with secondary antibodies at $37^{\circ} \mathrm{C}$ for $2 \mathrm{~h}$. The cover-slips were finally mounted on glass slides. The primary antibodies were as follows: Monoclonal mouse anti-pan-cytokeratin (CK) (cat. no. JY-0077, Jiayuan Quantum Dots Co. Ltd. dilution 1/25 for MCF-7 cells, 1/50 for SW480 cells, 1/25 for SGC7901 cells) and rabbit anti-Ki67 (JY-0047, Jiayuan Quantum Dots Co. Ltd. dilution 1/100 for MCF-7 cells, 1/250 for SW480 cells, 1/100 for SGC7901 cells). The secondary antibodies were QDs-525 goat $\mathrm{F}\left(\mathrm{ab} \mathrm{b}^{\prime}\right) 2$ anti-mouse IgG conjugate (Invitrogen, Thermo Fisher Scientific, Inc., Waltham, MA, USA; dilution 1/200, emitting green light at wavelength $525 \mathrm{~nm}$ ), QDs-585 goat F (ab') 2 anti-rabbit IgG conjugate (Invitrogen, Thermo Fisher Scientific, Inc.; dilution 1/200, emitting yellow light at wavelength $585 \mathrm{~nm}$ ) and QDs-605 goat F (ab') 2 anti-rabbit IgG conjugate (Jiayuan, China; dilution 1/100, emitting red light at wavelength $605 \mathrm{~nm}$ ).

Image acquisition and evaluation. The QD-stained slides were observed under an Olympus BX51 fluorescence microscope equipped with an Olympus DP72 camera (Olympus Optical Co., Ltd., Tokyo, Japan) and CRi Nuance multi-spectral imaging system (Cambridge Research \& Instrumentation, Inc., Woburn, MA, USA), at the excitation wavelength of 330-385 $\mathrm{nm}$ by ultraviolet light. A spectral cube for each slide was captured by CRi Nuance systems under the same conditions at low magnification (x20). The QD fluorescent signal for each cube was analyzed by CRi Nuance software package according to the manufacturer's instructions. The quantified fluorescence signals of Ki67 and pan-CK were calculated based on spectral unmixing. The ratio of Ki67 total fluorescence signal values to pan-CK total fluorescence signal values was regarded as the average expression intensity of Ki67. The quantified fluorescence signals of pan-CK were used to define the total number of cancer cells in clones. The clones were divided into three types based on the cell number in each clone, including small clones containing 14-49 cells, medium clones containing 50-100 cells, and large clones containing $>100$ cells.

Statistical analysis. Values are expressed as the medium for the average expression intensity of Ki67 in three types of cancer cells and in different sized clones, and a $\chi^{2}$ test was used for statistical analysis by SPSS 19.0 software package (IBM, Armonk, NY, USA). P $<0.05$ was considered to indicate a statistically significant difference.

\section{Results}

Cell clone formation assay. After cell culture on days 6,8 and 12, the MCF-7, SW480 and SGC7901 cells formed visible clones. According to the definition that cell cluster size $>50 \mu \mathrm{m}$ in diameter could be considered as a cell clone (18), a total of 119,123 and 120 clones were identified for MCF-7, SW480 and SGC7901 cells, respectively.

Heterogeneous morphology of cell clones. As shown in Fig. 1, multiple morphological features of cells in different clones could be observed. The MCF-7 cells in clones were spindle, triangular and various other irregular shapes (Fig. 1A). SW480 cells appeared as spindle, circular and irregular shapes (Fig. 1B), and SGC7901 cells were polygonal and spindle-shaped (Fig. 1C). These three types of cells had heterogeneous morphologies and cells ranged in size. Large cells were always located in the center or on one side of the clones, whereas, the cells at the periphery of the clones were smaller and were actively dividing. It was common to observe large nuclear:cytoplasmic ratios, pathological mitotic features, and multiple nuclei and nucleoli. Cells at the clone periphery were also observed to form several outstretching pseudopodia.

Discrete tendency of cell clones. In the majority of the cell clones, the peripheral cancer cells exhibited outstretched pseudopodia, whereas the remaining cells tended to deviate from the cancer cell clones and proliferate separately (Fig. 2).

Ki67 expression and distribution in cell clones. CRi Nuance multi-spectral imaging system was used to obtain fluorescent signals of QDs, in which, yellow or red fluorescent signals represented the Ki67 expression in the cell nucleus. Ki67 
A

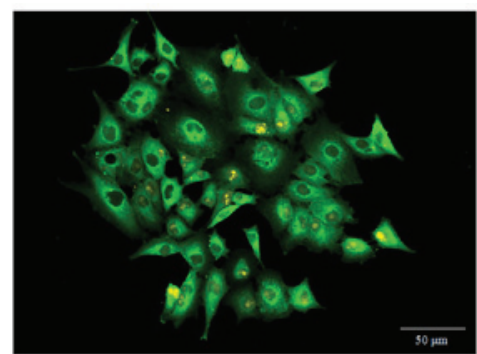

B

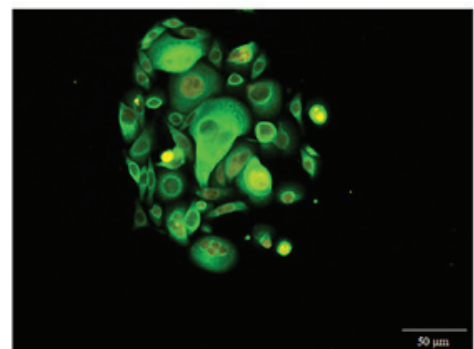

$\mathbf{C}$

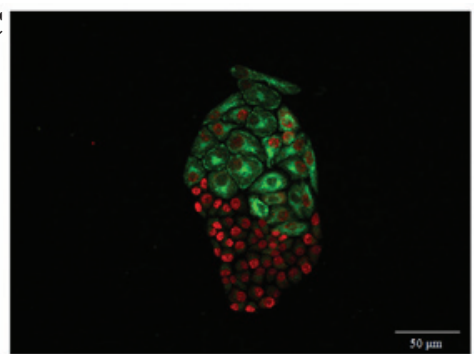

Figure 1. Morphological characteristics of cancer cell clones. (A) MCF-7 breast cancer cells were shuttle, triangle or irregular in shape. (B) SW480 colon cancer cells were shuttle, circular or irregular in shape. Magnification 200x, Goat anti-rabbit IgG-585 of yellow QDs marked Ki67. (C) SGC7901 gastric cancer cells were polygonal or shuttle in shapes. x200, Goat anti-rabbit IgG-605 of red QDs marked Ki67. Scale bar=50 $\mu \mathrm{m}$.

A

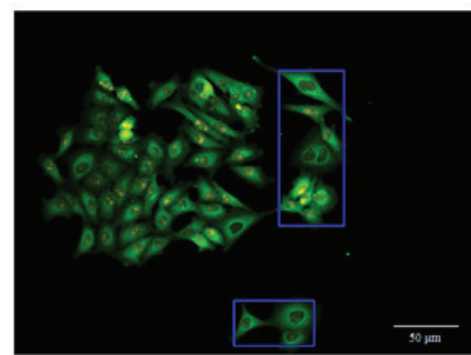

B

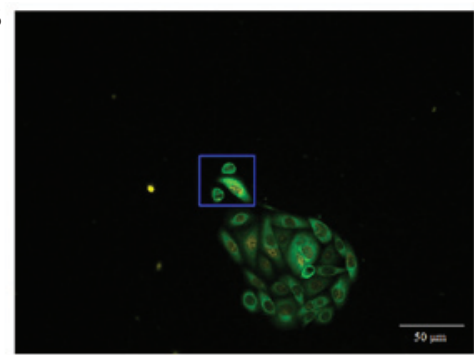

C

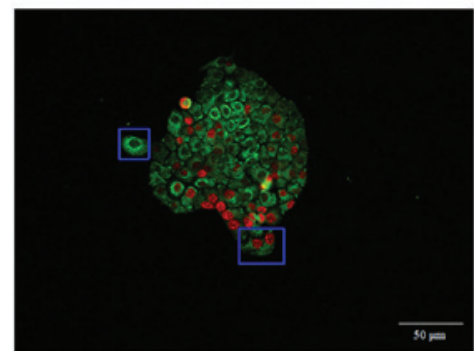

Figure 2. Discrete tendency of border cancer cells of the clones. The cells in blue box indicate the border cancer cells with outstretching pseudopodia in (A) MCF-7 breast cancer cells, (B) SW480 colon cancer cells and (C) SGC7901 gastric cancer cells. A and B: x200, Goat anti-rabbit IgG-585 of yellow QDs marked Ki67; C: x200, Goat anti-rabbit IgG-605 of red QDs marked Ki67. Scale bar=50 $\mu \mathrm{m}$.

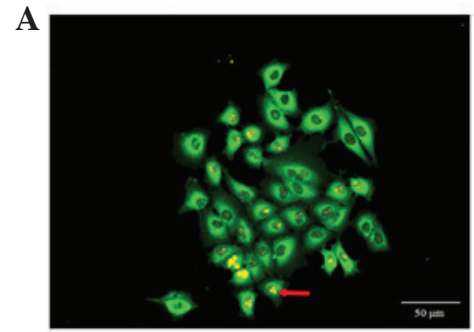

D

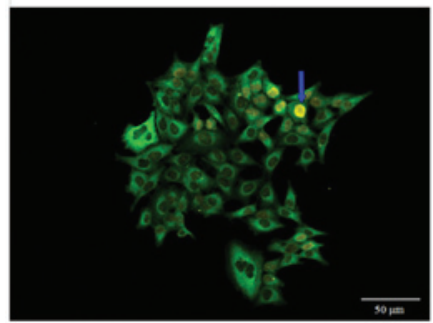

B

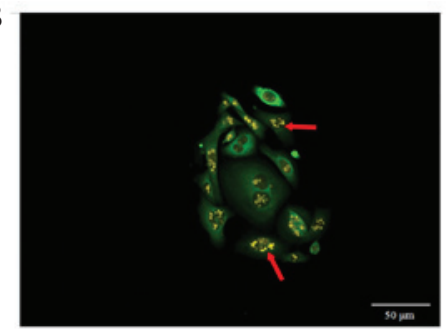

$\mathbf{E}$

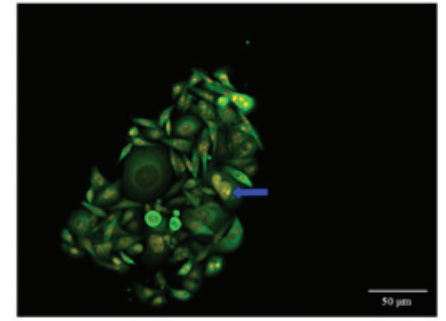

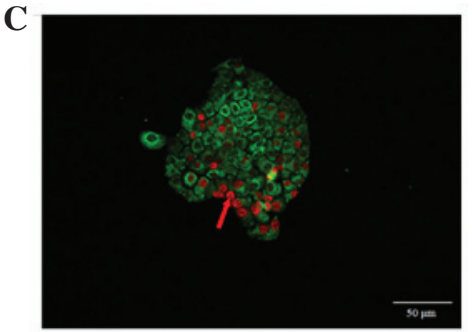

$\mathbf{F}$

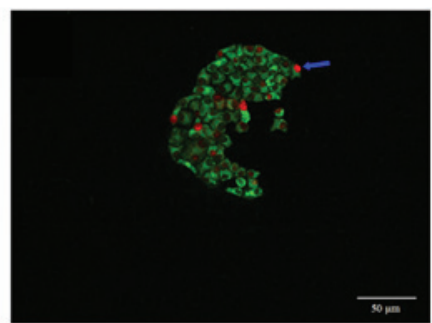

Figure 3. Ki67 expression and distribution in different cell clones. (A) Different sized clumps located on one side of the cell nucleus in MCF-7 breast cancer cells. (B) Different sized clumps evenly-distributed in cell nucleus in SW480 colon cancer cell. (C) Different sizes of clumps evenly-distributed in cell nucleus in SGC7901 gastric cancer cell. Homogeneous distribution of Ki67 was observed in the in cell nuclei of the (D) MCF-7 breast cancer cells, (E) SW480 colon cancer cells, and (F) SGC7901 gastric cancer cells. Red arrows indicated different sizes of clumps and blue arrows indicated even-distributed of Ki67 in the cell nucleus. (A and B: x200, Goat anti-rabbit IgG-585 of yellow QDs marked Ki67; C: x200, Goat anti-rabbit IgG-605 of red QDs marked Ki67. Scale bar $=50 \mu \mathrm{m})$.

expression in MCF-7 cells showed different sizes of clumps, predominantly on one side of cell nucleus (Fig. 3A). In the majority of the SW480 cells (Fig. 3B) and SGC7901 cells (Fig. 3C), Ki67 protein tended to form clumps, which were evenly distributed in the cell nucleus. Ki67 expression in these three types of cells also showed homogeneous distribution in the cell nucleus (Fig. 3D-F).
Distribution of cells with strong Ki67 expression. Ki67 was widely expressed in the three cancer cell types. The cells with strong Ki67 expression were distributed at the periphery of the clones or on one side of cell clones (Fig. 4). These cells presented two types of distribution, dispersed and aggregated, the second type forming cell clusters. However, there were only a small number of clones in which all cells showed 

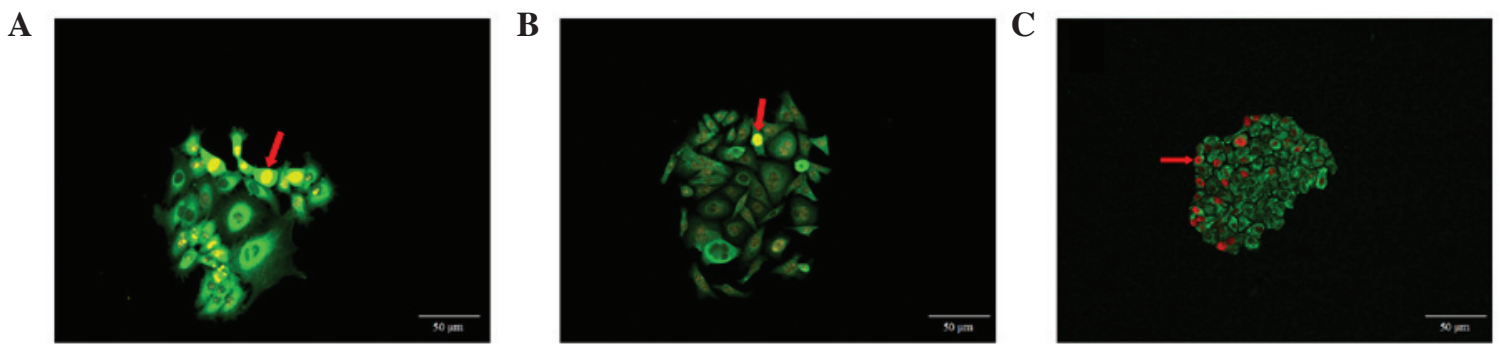

Figure 4. Heterogeneous distribution of cells with strong Ki67 expression. (A) Cells with strong Ki67 expression distributed at the periphery of MCF-7 cancer cell clones. (B) Cells with strong Ki67 expression were distributed at one side of the SW480 cell clones. (C) Cells with strong Ki67 expression were distributed at one side of the SGC7901 cell clones. Red arrows indicate the cancer cells with strong Ki67 expression. (A and B: x200, Goat anti-rabbit IgG-585 of yellow QDs marked Ki67; C: x200, Goat anti-rabbit IgG-605 of red QDs marked Ki67. Scale bar=50 $\mu \mathrm{m}$ ).

A

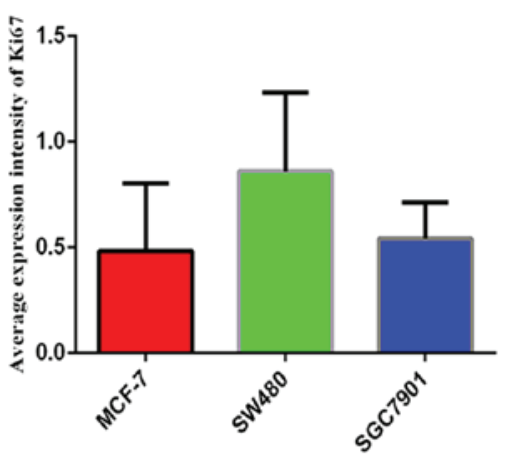

C

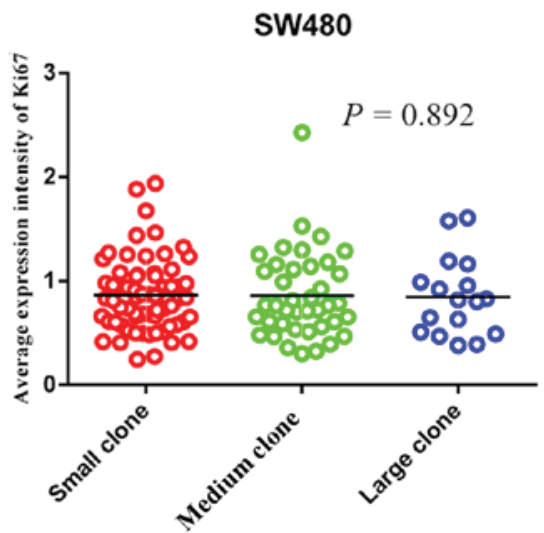

B

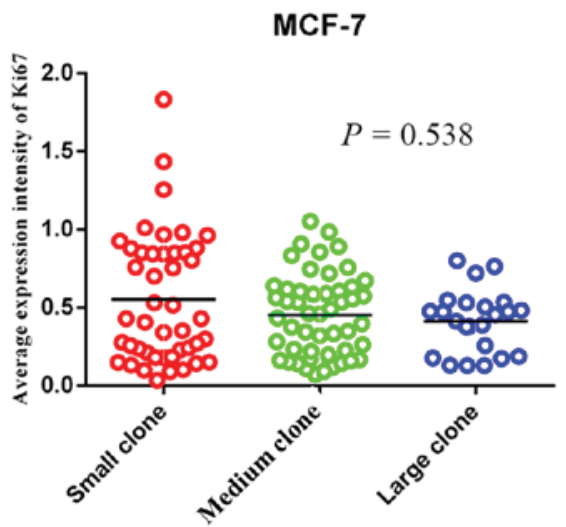

D

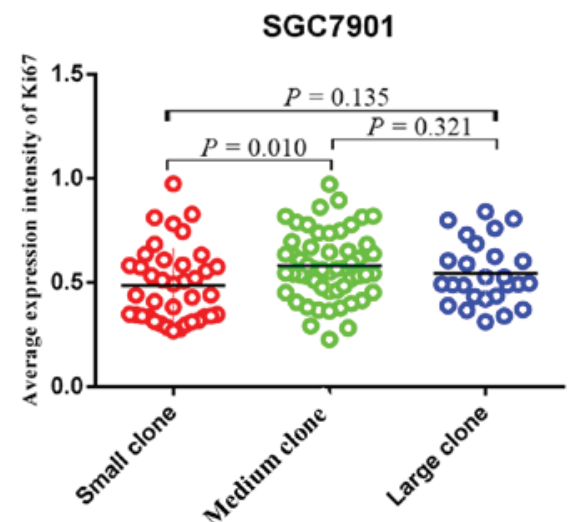

Figure 5. Average expression intensity of Ki67 in three types of cancer cells and in different sizes of clones. (A) Average expression intensity of Ki67 in different sized clones of MCF-7 cells. (B) Average expression intensity of Ki67 in different sizes of clones of SW480 cells. (C) Average expression intensity of Ki67 in different sized clones of SGC7901 cells. (D) Average expression intensity of Ki67 in these three types of cancer cells.

strong Ki67 expression. These results demonstrate the asymmetry and asynchronism of cell proliferation in cell clones, with a portion of dominant cells showing active proliferation potential.

Average expression intensity of Ki67 in the three cancer cell types. According to the statistical analysis, the mean expression intensity of Ki67 in MCF-7, SW480 and SGC7901 cells was $0.433(0.036,1.833), 0.810(0.246,2.428)$ and $0.532(0.227$, 0.974 ), respectively (Fig. 5A). The mean expression intensity of Ki67 in small, medium and large clones of MCF-7 cells was $0.429(0.036,1.833), 0.459(0.072,1.052)$ and $0.455(0.128$, $0.801)$, no significant difference was identified among the three groups ( $\mathrm{P}>0.05$; Fig. 5B). For SW480 cells, the corresponding values were $0.836(0.246,1.939), 0.764(0.301,2.428)$ and $0.820(0.381,1.608)$, again no significant difference was identified among the three groups ( $\mathrm{P}>0.05$; Fig. $5 \mathrm{C})$. By contrast in SGC7901 cells, the mean expression intensity of Ki67 in small, medium, large clones were $0.440(0.268,0.974), 0.562$ $(0.227,0.972)$ and $0.512(0.312,0.840)$, and a significant difference was identified between the expression in the small and medium clones ( $\mathrm{P}<0.05$; Fig. 5D). 


\section{Discussion}

Clone formation assays are a widely used technique to investigate cell proliferation and invasion (19). QDs are novel fluorescent nano-particles, which have shown great potential in diagnosis and treatment, for example in vivo and in vitro imaging and drug delivery (20-22). In the present study, a cell clone formation assay was applied to simulate tumor development and progression in vitro, and this was analyzed using proliferating cell nuclear antigen Ki67 and pan-CK marked by different QD-conjugated probes. With the aid of QD-based molecular targeted imaging techniques, cell clone formation could be used to demonstrate the distribution and expression of Ki67 in different types of cancer cells, and reveal the clonal growth behavior and proliferation characteristics of the cancer cells.

Traditional double-color fluorescent imaging techniques mark different cellular components separately and use image merging technology to compose a full image of the cell; however, this can not implement in situ, simultaneous or synchronous images of multiple cellular components, leading to errors in the integrity and completeness of cell image information $(23,24)$. By contrast, the present study took advantage of the in situ, synchronous, double-color imaging technique which utilizes QD-based molecular probes. It was able to capture the morphology of cancer cells in situ and simultaneously revealed Ki67 expression and distribution in the nucleus and pan-CK expression in the cytoplasm. Furthermore, this information could be analyzed under CRi Nuance multi-spectral imaging systems to output the quantitative data of Ki67 and pan-CK expression in cancer cell clones, which indicated the effects of proliferation behavior of each type of cancer cell during the formation and development of whole clones.

$\mathrm{Ki67}$, a cell-cycle-related non-histone, is expressed at all cell cycle phases except for the $\mathrm{G}_{0}$ phase (5). In this study, Ki67 protein tended to form clumps in MCF-7 cells, which were evenly distributed in the cell nucleuss, predominantly located on one side of the cell nucleus. In the majority of the SW480 and SGC7901 cells, Ki67 presented different sizes of clumps evenly distributed in the cell nucleus, which is consistent with the results of Scholzen and Gerdes (5), which demonstrated that Ki67 formed clumps during interphase, and was evenly distributed in the nucleus during mitosis. Cells to undergo mitosis had higher Ki67 expression levels. In addition, the tumor proliferation index (25), a traditional index of clinical pathology, could be represented by the ratio of Ki67 positive cells to the total number of cancer cells. In this study, the ratio of Ki67 total fluorescence signal values to pan-CK total fluorescence signal values was regarded as the average Ki67 expression intensity, which was $0.433(0.036,1.833), 0.810$ $(0.246,2.428)$ and $0.532(0.227,0.974)$, respectively, in MCF-7, SW480 and SGC7901 cells. The average Ki67 expression intensity was highest in the SW480 cells and Ki67-positive cells were undergoing mitosis according to the research of Scholzen and Gerdes (5). SGC7901 cells had the second highest intensity level. The MCF-7 cells had the lowest intensity level, which indicated the Ki67 positive cells in MCF-7 cells clones were primarily in the interphase stage of cell division. These points indicated the average expression intensity of Ki67 could reflect cancer cell division and proliferation, and could be considered as a proliferation index of cancer cells.
Brabletz et al (26) reported that colon cancer cells may exhibit cell de-differentiation in the invasive front area, with loss of an epithelial phenotype and the gain of a mesenchymal phenotype, which could facilitate the invasion and metastasis of tumor cells. Numerous other tumors also showed these characteristics (26-28). The over-expression of nuclear Ki67 in the invasive front area in breast cancer was positively associated with bone and liver metastasis (29). In the present study, in these three types of cancer cell clones, the cells with strong Ki67 expression were distributed at the clone periphery or at one side of the cell clones. In addition, in the majority of the cell clones, the cancer cells with outstretching pseudopodia were separated from clones or showed a tendency of discretion. These phenomena indicated that the cancer cells at the clone periphery exhibit greater proliferative and invasive activity. Thus, cell clone formation assays was an efficient method to simultaneously study proliferative and invasive characteristics of tumor cells.

In conclusion, this study adopted a QD-based molecular targeted imaging technique, simultaneously showing the morphological characteristics of cancer cells and cell proliferation in the process of cancer cell clone formation in situ, which demonstrated that cancer cell proliferation was asymmetric and unsynchronized. The phenomenon that cancer cells within clones that had heterogeneity determined that cancer cells had uncontrollable and in-coordinate proliferative features (30). Additionally, this suggested that there is theoretical feasibility and technical possibility to develop a differentiation strategy which may allow for the control and coordination of cancer cells.

\section{Acknowledgements}

This study was supported by the Key Project of Natural Science Foundation of China (grant no. 81230031/H18), the Youth Project of Natural Science Foundation of China (grant no. 8140110827/H1606) and the Key Project of the Fundamental Research Fund for the Central Universities (grant no. 303274028).

\section{References}

1. Dowsett M, Nielsen TO, A'Hern R, Bartlett J, Coombes RC, Cuzick J, Ellis M, Henry NL, Hugh JC, Lively T, et al: Assessment of Ki67 in breast cancer: Recommendations from the international Ki67 in breast cancer working group. J Natl Cancer Inst 103: 1656-1664, 2011.

2. Soltysova A, Altanerova V and Altaner C: Cancer stem cells. Neoplasma 52: 435-440, 2005.

3. Franken NA, Rodermond HM, Stap J, Haveman J and van Bree C: Clonogenic assay of cells in vitro. Nat Protoc 1: 2315-2319, 2006.

4. Munshi A, Hobbs M and Meyn RE: Clonogenic cell survival assay. Methods Mol Med 110: 21-28, 2005.

5. Scholzen T and Gerdes J: The Ki-67 protein: From the known and the unknown. J Cell Physiol 182: 311-322, 2000.

6. He X, Chen Z, Fu T, Jin X, Yu T, Liang Y, Zhao X and Huang $\mathrm{L}$ : Ki-67 is a valuable prognostic predictor of lymphoma but its utility varies in lymphoma subtypes: evidence from a systematic meta-analysis. BMC Cancer 14: 153, 2014.

7. Wang LW, Qu AP, Liu WL, Chen JM, Yuan JP, Wu H, Li Y and Liu J: Quantum dots-based double imaging combined with organic dye imaging to establish an automatic computerized method for cancer Ki67 measurement. Sci Rep 6: 20564, 2016.

8. Weng KC, Nobel CO, Papahadjopolous-Sternberg B, Chen FF, Drummond DC, Kirpotin DB, Wang D, Hom YK, Hann B and Park JW: Targeted tumor cell internalization and imaging of multifunctional quantum dot-conjugated immunoposomes in vitro and in vivo. Nano Lett 8: 2851-2857, 2008. 
9. Gokarna A, Jin LH, Hwang JS, Cho YH, Lim YT, Chung BH, Youn SH, Choi DS and Lim JH: Quantum dot-based protein micro- and nanarrays for detection of prostate cancer biomarkers. Proteomics 8: 1809-1818, 2008.

10. Chen LD, Liu J, Yu XF, He M, Pei XF, Tang ZY, Wang QQ, Pang DW and Li Y: The biocompatibility of quantum dot probes used for the targeted imaging of hepatocellular carcinoma metastasis. Biomaterials 29: 4170-4176, 2008.

11. Chen C, Xia HS, Gong YP, Peng J, Peng CW, Hu MB, Zhu XB, Pang DW, Sun SR and Li Y: The quantitative detection of total HER 2 load by quantum dots and the identification of a new subtype of breast cancer with different 5-year prognosis. Biomaterials 31: 8818-8825, 2010.

12. Peng CW, Liu XL, Chen C, Liu X, Yang XQ, Pang DW, Zhu XB and Li Y: Patterns of cancer invasion revealed by QDs-based quantitative multiplexed imaging of tumor microenvironment. Biomaterials 32: 2907-2917, 2011.

13. Hamburger AW and Salmon SE: Primary bioassay of human tumor stem cells. Science 197: 461-463, 1997.

14. Chen C, Peng J, Xia HS, Yang GF, Wu QS, Chen LD, Zeng LB, Zhang ZL, Pang DW and Li Y: Quantum dots-based immunofluorescence technology for the quantitative determination of HER2 expression in breast cancer. Biomaterials 30: 2912-2918, 2009.

15. Chen C, Sun SR, Gong YP, Qi CB, Peng CW, Yang XQ, Liu SP, Peng J, Zhu S, Hu MB, et al: Quantum dots-based molecular classification of breast cancer by quantitative spectroanalysis of hormone receptors and HER2. Biomaterials 32: 7592-7599, 2011

16. Fang M, Yuan JP, Peng CW, Pang DW and Li Y: Quantum dots-based in situ molecular imaging of dynamic changes of collagen IV during cancer invasion. Biomaterials 34: 8708-8717, 2013.

17. Wang FB, Rong Y, Fang M, Yuan JP, Peng CW, Liu SP and Li Y: Recognition and capture of metastatic hepatocellular carcinoma cells using aptamer-conjugated quantum dots and magnetic particles. Biomaterials 34: 3816-3827, 2013

18. Li X, Pan Y, Fan R, Jin H, Han S, Liu J, Wu K and Fan D: Adenovirus-delivered CIAPIN1 small interfering RNA inhibits HCC growth in vitro and in vivo. Carcinogenesis 29 1587-1593, 2008.
19. Plumb JA: Cell sensitivity assays: Clonogenic assay. Methods Mol Med 88: 159-164, 2004.

20. Frangioni JV: New technologies for human cancer imaging. J Clin Oncol 26: 4012-4021, 2008.

21. Kim J, Piao Y and Hyeon T: Multifunctional nanostructured materials for multimodal imaging, and simultaneous imaging and therapy. Chem Soc Rev 38: 372-390, 2009.

22. Hong H, Zhang Y, Sun J and Cai W: Molecular imaging and therapy of cancer with radiolabeled nanoparticles. Nano Today 4: 399-413, 2009.

23. Peng CW, Tian Q, Yang GF, Fang M, Zhang ZL, Peng J, Li Y and Pang DW: Quantum-dots based simultaneous detection of multiple biomarkers of tumor stromal features to predict clinical outcomes in gastric cancer. Biomaterials 33: 5742-5752, 2012.

24. Resch-Genger U, Grabolle M, Cavaliere-Jaricot S, Nitschke R and Nann T: Quantum dots versus organic dyes as fluorescent labels. Nat Methods 5: 763-775, 2008

25. Beresford MJ, Wilson GD and Makris A: Measuring proliferation in breast cancer: Practicalities and applications. Breast Cancer Res 8: 216, 2006.

26. Brabletz T, Jung A, Spaderna S, Hlubek F and Kirchner T: Opinion: Migrating cancer stem cells-an integrated concept of malignant tumour progression. Nat Rev Cancer 5: 744-749, 2005.

27. Watanabe T, Takahashi A, Suzuki K, Kurusu-Kanno M, Yamaguchi K, Fujiki H and Suganuma M: Epithelial-mesenchymal transition in human gastric cancer cell lines induced by TNF- $\alpha$-inducing protein of Helicobacter pylori. Int J Cancer 134: 2373-2382, 2013

28. Fang M, Yuan J, Peng C and Li Y: Collagen as a double-edged sword in tumor progression. Tumour Biol 35: 2871-2882, 2014.

29. Gong P, Wang Y, Liu G, Zhang J and Wang Z: New insight into Ki67 expression at the invasive front in breast cancer. PLoS One 8: e54912, 2013.

30. Fidler IJ: The pathogenesis of cancer metastasis: The 'seed and soil' hypothesis revisited. Nat Rev Cancer 3: 453-458, 2003. 